\title{
A Complex Systems Modelling Approach for Decentralized Simulation of Electrical Microgrids
}

\author{
Enrique Kremers and Pablo Viejo \\ European Institute for Energy Research - EIFER \\ EDF and the University of Karlsruhe \\ Karlsruhe, Germany. \\ Email: enrique.kremers@eifer.uni-karlsruhe.de
}

\author{
Oscar Barambones and José González de Durana \\ University College of Engineering \\ University of the Basque Country \\ Vitoria-Gastez, Spain \\ Email: josemaria.gonzalezdedurana@ehu.es
}

\begin{abstract}
The structure and behavior of Electrical Grids share many of the properties of Complex Computer Systems, with microgrids and other decentralized electrical systems attached to them, so they can be interpreted as Systems of Systems. Furthermore, the evolution of the future electrical system will bring a higher degree of decentralization concerning specially production and control. To deal with this paradigm change, new models and tools are necessary. In this paper a model of an electrical microgrid is presented. The approach used in the development of the model is Agent-Based in combination with System Dynamics. By mixing these approaches the different entities of the electrical system (production, demand, storage, etc.) have been represented. Through the individual behavior of the agents it is possible to reproduce the complex behavior of the system as a whole. This behavior can produce expected and unexpected emergent properties on the interconnected system that can be analyzed through simulation. A case study is presented to analyze the capabilities of such kind of models. The example shows the simulation of an integrated microgrid system, where different components such as renewable energy sources and storage have been implemented. The simulation results of this case study are discussed.
\end{abstract}

Index Terms-electrical grid, microgrid, agent-based models, complex computer systems, system of systems.

\section{INTRODUCTION}

The introduction of renewable energy carriers in developed countries implies one of the greatest changes in the structure of their energy systems. These systems are moving away from a centralized and hierarchical energy system, where the production follows a top-down principle under strict control of the electricity supply companies, towards a new system where distributed actors influence the energy supply. Production is not limited any more to large energy providers, as small decentralized producers enter the network and inject energy at much lower tension levels than before.

\section{MICROGRIDS}

A microgrid is an integrated energy system consisting of interconnected loads and distributed energy resources which as an integrated system can operate in parallel with the grid or in an intentional island mode [2]. A microgrid is as well a set of small energy generators arranged in order to supply energy for a community of users in close proximity. It is a combination of generation sources, loads and energy storage, interfaced through fast acting power electronics. In the course of this paper we argue how an the electric grid and also a microgrid can be understood as a "Complex Computer System".

Microgrids represent a form of decentralization of electrical networks. They comprise low or medium voltage distribution systems with distributed energy sources, storage devices and controllable loads.

The European Commission [4] defines microgrids as small electrical distribution systems that connect multiple customers to multiple distributed sources of generation and storage. It also described that microgrids typically can provide power to communities up to 500 households at low voltage level. This shows that this technology is not only suitable for electrification of small remote islands or remote zones in developing countries but for the integration into existing high voltage transmission grids, too. Therefore microgrids can have a wide application spectrum in near future.

During disturbances, the generation and corresponding loads can autonomously disconnect from the distribution system to isolate the load of the microgrid from the disturbance without damaging the integrity of the transmission grid.

From the point of view of the customer, it can be seen as a low voltage distribution service with additional features like increase in local reliability, improvement of voltage and power quality, reduction of emissions, decrease in cost of energy supply, etc.

A microgrid is connected to the distribution network through a single point of common coupling (PCC) and appears to the power network as a single unit.

A number of authors argue that power electronics would be a crucial feature regarding microgrids since most of the power microsources must be electronically controlled to gain the required characteristics for the system.

1) Microgrid architecture: A report by Navigant Consulting [2] prepared for DOE's Office of Electricity Delivery and Energy Reliability identifies four classes of Microgrids:

- Single Facility Microgrids

These Microgrids include installations such as industrial and commercial buildings, residential buildings, and hospitals, with loads typically under $2 \mathrm{MW}$.

- Multiple Facility Microgrids

This category includes Microgrids spanning multiple buildings or structures, with loads typically ranging be- 


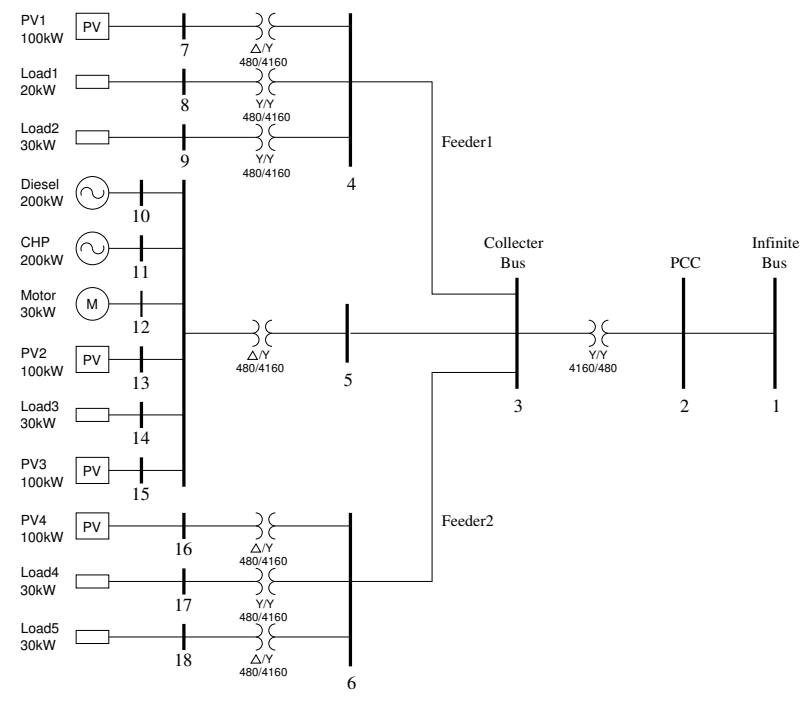

Fig. 1. Microgrid schemme

tween 2 and 5 MW. Examples include campuses (medical, academic, municipal, etc), military bases, industrial and commercial complexes, and building residential developments.

- Feeder Microgrids

The feeder Microgrid manages the generation and/or load of all entities within a distribution feeder, which can encompass 5-10MW. These Microgrids may incorporate smaller Microgrids - single or multiple facility- within them.

- Substation Microgrids

The substation Microgrid manages the generation and/or load of all entities connected to a distribution substation, which can encompass 5-10+MW.

Figure 1 shows a Multiple Facility Microgrid, with two integrated Feeder Microgrids.

2) Microgrid control: Concerning the control of microgrids, direct control over local distribution and transmission of electricity from the interconnect point across a facility is a very common practice.

Electricity grids are becoming intelligent, true complex computer systems, with flexible, controlled power flows supported by advanced information technology [6]. Under the new smart grid paradigm emerged in the last decade, new capabilities for measurement, control and communication, with a two-way flow of energy and information between customer and supplier, are demanded, in order to rise efficiency and get a cleaner electricity generation.

Some of such new operational measurement and control capabilities that are needed to manage smart grids are:

- Network based communication and control

- Monitor energy generation and consumption

- Optimization of production and consumption

- Generation and load dispatching

- Energy production and consumption control

- Tieline control
- Microgrid active and reactive power control

- Microgrid connection and islanding control

- Security standards

- User-based billing

Some of the key technical issues not entirely solved at present are

- Real time power flow balancing

- Voltage control and security during disconnection (islanding) from the point of common coupling (PCC)

- Failures protocol response

- Protection and stability aspects

- Dynamic short term and long term response

- Active and reactive power control

- Communication technologies adaptation

- Tieline control

On these points, research is still required and so modelling and simulation of microgrids is an unavoidable instrument on the way to an efficient and secure physical implementation.

\section{MODELS}

For modeling electrical grids, it is necessary to use new design tools (different to the traditionally used in Electrical Engineering) and agent based software able to capture the complex system behavior, the wanted and unwanted emergent behavior, the internal and external events, the system communications, etc. Simulation can be very helpful for designing electrical grids and microgrids, because while a lot of intelligent network components do not exist yet, they can be modeled and used in the simulation. Therefore, it is possible to use components before their market deployment in the simulation and future designs of intelligent networks can be tested.

The analysis of system architectures can be a relevant first step for future grid development. Despite the results of the simulation may fit for some ideal architectures, the development of "enabling" technologies is necessary. Some of them can be already available in the market or being employed in other sectors, but probably other are completely new or still far away from commercialization and widespread deployment. While waiting for research, development and commercialization of such elements, simulation can be very useful to analyze the capabilities of future systems.

\section{A. The electrical grid as a Complex Computer System and System of Systems}

The electrical grid can be considered as a true Complex Computer System whose aim is to manage power flows. Indeed it consists of a set of vertices (nodes or buses with a computer) with two types of edges arranged between them: the first one (power lines) is dedicated to carry out the power flow and the second one (information channels) to broadcast the information flow. In the authors opinion, the assumption that a computer is placed on each node does not appear to involve a great loss of generality to the model. This artificial connection precisely defines the "outside view" or environment of the 


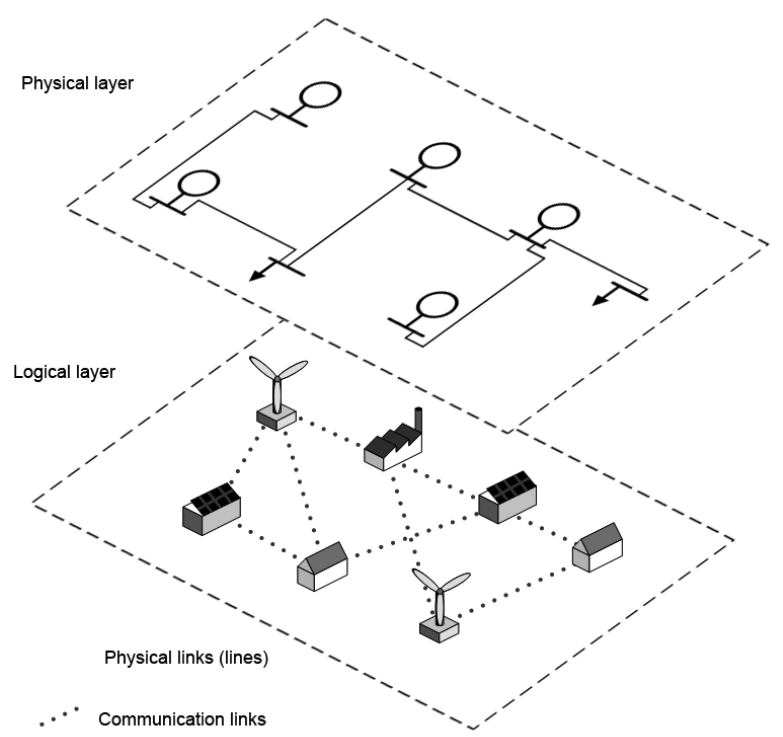

Fig. 2. Different layers in an electrical microgrid

system. Therefore, the electrical system can be split in two layers:

- Physical layer: The first layer comprehends the physical structure of the electrical grid itself, including all the power transmission lines. It includes the power flows as wells as all the electrical parameters concerning the correct operation of the grid.

- Logical layer: This second layer, which represents the main part the upcoming generation of electrical grids is not yet present in contrast to the physical layer. This layer includes all the information exchange that has to be arranged to control DG, dispatchable loads and all other smart equipment in future grids. It has to be underlined that the communication paths have not to be the same than the links in the first layer, although they could be exploited for that aim (Power line communication, PLC).

Concerning the second layer, in this work a specific information and communication technology is intentionally do not fixed, it is rather treated from the theoretic point of view. The specifications of this layer include a real time communication between the elements of the grid. In [3] an Internet of Energy is suggested as a parallelism to computer networks. Particularly this medium could itself serve as a communication platform. More examples for the implementation of the logical layer could be:

- Wired-based technologies

- Using the grid infrastructure: Power line communication

- Other existing or new wired networks such as the Internet or other cable networks

- Wireless technologies, such as WLAN or WMAN

Also geographic network localisation, distribution of processing and databases, interaction with humans, and unpre-

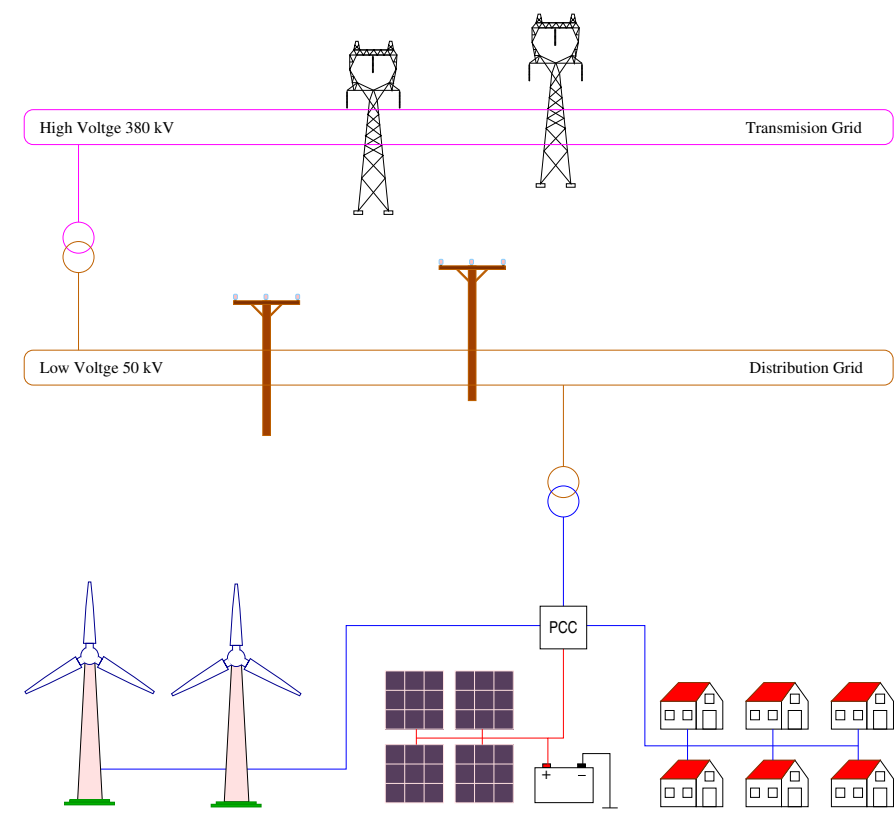

Fig. 3. Integrated microgrid

dictability of system reactions to unexpected external events are present in this kind of networks.

Figure 3 shows a schematic drawing of an integrated microgrid, showing the point of common coupling (PCC), but without the information channels.

One can easily argue that the electrical grid, and also a microgrid, (nearly) satisfy the five principal characteristics that distinguish them as true systems-of-systems [5].

1) Operational Independence of the Elements: Electrical grid (and microgrid) systems are able to usefully operate independently. So generators, loads and other elements work properly when they are not connected to the grid.

2) Managerial Independence of the Elements: The electrical grid (and microgrid) systems not only can operate independently, they do operate independently. They maintain a continuing operational existence independent of the system-of-systems.

3) Evolutionary Development: The electrical grid (and microgrid) does not appear fully formed. Its future development and existence is evolutionary and depends of other environmental agents.

4) Emergent Behavior: The electrical grid (and microgrid) performs functions and carries out purposes that do not reside in any component system. These behaviors are emergent properties of the entire system-of-systems and cannot be localized to any component system. The main goals of electrical grid and microgrid are given by these behaviors.

5) Geographic Distribution: The geographic extent of the electrical grid is large and also microgrids can be of some extension. The electrical grid components can readily exchange information (but in this case they can also exchange energy). 


\section{APPROACH}

A model of a "Microgrid" implemented in AnyLogic is presented below. The model is completely open so it can be used to address a number of issues of design and computation that arise in such networks, some of which are:

- Grid and microgrid simulation, control, being basis for other models

- Dynamic power flow computing

- Loads power consumption, load dispatching, optimize energy consumption, generation and storage, collect and report

- Grid and microgrid architecture design

- Centralized and decentralized control design

- Load connection and disconnection

- Microgrid connection and islanding modes

- Branch or node (substation) deleting

- Microgrid optimal design

- Economic benefits demonstration

The approach presented in the following is intended to obtain a suitable electrical microgrid model. It is a continuation of the authors previous work in this area [11], [12]. In these works, the mesh method was used to obtain voltages at the mesh nodes and currents through the branches, assuming known voltages given by generators. In the present work though a new power flow method has been built. An important part of the work has been to adapt or rather redesign the old power flow method to the dynamic case, using an agent based method, in which charges, voltages in generators, and even the structure of the grid, may change.

This new method has been implemented with the Anylogic's Agent Based paradigm, using agents for representing the buses of the grid.

\section{A. AnyLogic modeling}

AnyLogic is a simulation program developed by $\mathrm{XjTek}$ [9] using a subset of UML for Real Time (UML-RT) as a modeling language, and extended it to incorporate continuous behavior.

Three simulation paradigms are included in AnyLogic:

- System Dynamics (SD)

- Discrete Event (DE)

- Agent Based (AB)

SD and DE are more traditional approaches whereas $\mathrm{AB}$ is relatively new. This methods can be combined in a model, for example it is possible to include SD and DE methods into an agent model, so each agent can be an hybrid system. With this kind of models a very high refinement level can be are obtained.

The Dynamic System (DS) paradigm, the main method used in block based programs like Matlab-Simulink, is considered to be as a part of SD paradigm in AnyLogic since version 6 appeared.

Although AnyLogic is specially well featured to model Hybrid Systems, like electric and electronic circuits, there are not many papers about modeling electrical networks nor power system generation and distribution made using this software [10].

\section{B. Power Flow analysis}

To design an electrical network engineers need to be able to estimate the voltages and currents at any places within the circuit. Usually an undirected graph shows the grid topology and mesh analysis or nodal analysis is often used to pose an equation system and solve it for the voltage and current at any place in the circuit. Standard lineal algebra methods are suitable for linear systems, but other non linear circuits only can be solved using estimation techniques or specialized software programs. The most widely used of all these is the Power Flow analysis. Summarized, the method can be described as follows.

In an electrical network with $n$ nodes, each one associated to a pair of complex numbers $V$ (voltage) and $S=P+Q i$ (apparent power)

The data is the following:

- $|V|$ and $\angle V=0$ are given at the reference node

- $|V|$ and $P$ are given at control nodes

- $P$ and $Q$ are given at load nodes (the other nodes)

Applying the electrical laws gives us a nonlinear system of $2 n$ equations with $2 n$ real unknowns

$$
g(x)=0
$$

where $x, g(x) \in \mathbb{R}^{2 n}$, with the unknowns:

- $P, Q$ for the reference or slack bus,

- $\angle V$ and $Q$ for control buses,

- $|V|, \angle V$ for load buses.

To solve the system, the most common approach in literature is to apply the Gauss-Jordan (iterative, of slow convergence) or the Newton-Raphson (faster, but requires to calculate the Jacobian matrix and invert it) method.

When talking about power flows, for example [1], it is often assumed that the electrical network is static. In fact it is assumed that all data values are known constants and the effect of actual load variations with time is studied by considering a number of different cases for which steady state conditions are assumed.

Even though, there are situations where after a value change in some nodes (e.g. when connecting or disconnecting loads and generators), the values of the unknowns have to be updated in a short time, because these values are used for the control of the network or for its simulation.

\section{Agent based modeling the electrical grid}

As said before, the traditional method used for the flow calculation of electrical network is based on static power flow calculations. These require the computation of a steady state of the network, and have to be rerun each time a change occurs if a continuous simulation wants to be executed. This can lay to a large number of re-calculations, leading to redundant steps, when only small changes affect small parts of the network. Implementing an agent based approach could try to solve these 
issues by providing more flexible and dynamic algorithms, and through the combination of traditional and agent based modeling techniques.

In the authors opinion, agent based modeling is specially well suited to cope with all difficulties one finds trying to obtain a dynamic model of the electrical grid. The proposed model use the three central ideas of this paradigm: agents to represent electrical buses, the environment object to represent in space and time the geographic area where the power lines are wired, and then single rules are bring up to each agent in order to induce the necessary complexity to the model. Then, emergent properties of the model will represent the electrical grid behavior [7].

Agent interactions are created at two levels (see 2). At the physical level they are given by electrical circuits laws (Ohm and Kirchhoff laws, etc.), translated to computational power flow algorithm rules. At the logical level, interactions represent internal and external events and they are implemented as agent messages in the model. In this way it is possible to create intelligent agents (buses) which are able to easily carry out some typical grid tasks as power flow control, generation sources dispatching, load dispatching, grid connection and disconnection, etc.

\section{CASE STUdY: AN INTEGRATED MICROGRID}

Hybrid Renewable Energy Systems include behaviors on different time scales, so the establishment of a single model covering all details would have as advantage the universality, but it also have some drawbacks, as for example the difficulty to understand the dominant behavior of the system and also, due the high complexity of the model, it would get an excessively long simulation time. The authors have developed

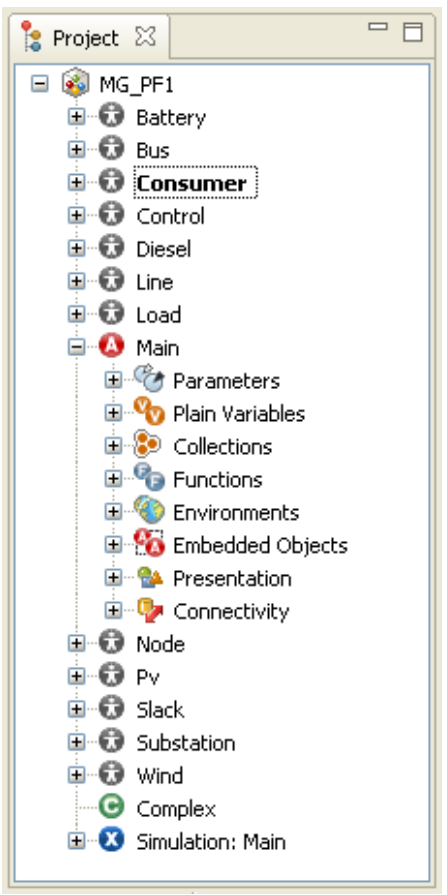

Fig. 4. Project view a computational hybrid, agent based, system modeling and simulation method, as a valuable tool for analysis, design and validation of micro-grids and, trying to simplify the exposition and the result analysis, and also for didactic reasons, the method is applied to a rather simple one.

The model has been built in AnyLogic using formulae given in [14] for the development of some of the components. It is composed of twelve Active Object Agent classes. Seven of them, Battery, Consumer, Diesel, Node, Substation, PV and Wind are intended to model the elements at the logical layer (see figure 2) and the other five, Bus, Slack, Control, Load and Line besides the Main class are for the physical layer elements. Figure 4 shows the AnyLogic Project window where the object tree can be seen.

The intention of this paper is no to describe all the elements in detail, but rather give an systemic view describing the key features of each agent.

The modeling of the microgrid consists in the integration of the power flow implementation in Anylogic into the "behavior" model of the agents. This is comparable to the two layers, the power flow symbolizing the physical flows, and the agent behavior the logical layer, i.e. the behavior of the agents. The power flow is performed at each time step, and computes the voltage angles and bus unknowns for all nodes. The time resolution chosen for calculation was in minutes as a first step but can be adapted to finer values, so that transient effects can be analyzed closer.

\section{A. The model of the microgrid}

The microgrid consists in a grid attached to a transmission network through a substation, as it can be seen in Figure 3 . The grid includes the following elements

- Diesel generator

- Wind power generator

- Photovoltaic element and Battery

- Two loads

- Substation

The photovoltaic panel is connected to the grid through an electronic inverter. The topology of the grid can be see in Figure 3. Each agent has an autonomous behavior, corresponding to its technical characteristics, intended or unintended random changes, etc. The model of each type of agent comprising the microgrid and their behavior that results therefrom are described in the following section.

1) Diesel generator: In the case the microgrid is turned on islanding mode and the load requirements are not met by either renewable energy system or by batteries, the power that is still required must be provided by the generator. The overall efficiency of a diesel generator is defined as follows:

$$
\eta_{\text {overall }}=\eta_{\text {brakethermal }} \cdot \eta_{\text {generator }} \text {, }
$$

where $\eta_{\text {brakethermal }}$ is the brake thermal efficiency of diesel engine. Normally, diesel generators are modeled to control hybrid power systems in order to achieve required autonomy. It can be observed that only high power operation mode (70$90 \%$ of full load) is efficient and economically reasonable. In 


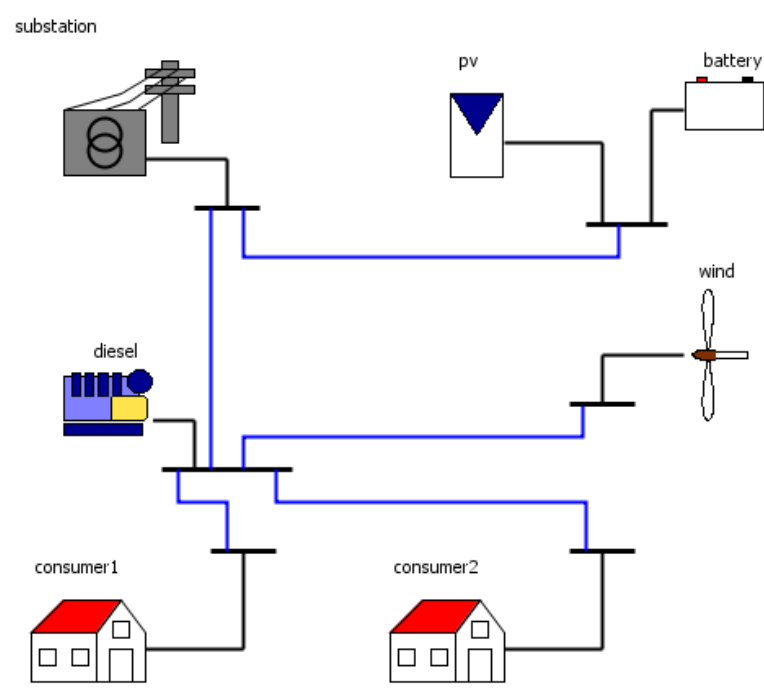

Fig. 5. Topology of the microgrid used in the case study

the absence of peak demand, the diesel generator can be used for battery charging, too. It has to be noted that the diesel generator here symbolizes a dispatchable unit that can supply energy in critical situations, but it could be also replaced by any other controllable source such as a (micro-)CHP, for example. The generator is modeled as a PV-bus.

2) Wind system simulation: Wind power is the renewable energy source (RES) that has achieved the greatest penetration rates in the last decades. Despite of being relatively economic and profitable and gaining a high popularity, wind power installations implicate a highly fluctuating generation and are difficult to predict. This is often a cause of grid stability problems. The model described here aims to consider the main points that characterize this generation source. The model can be split into two parts, the wind turbine generator itself and the simulation model for the wind speed.

The power output of wind turbine generator at a specific site depends on wind speed at hub height and speed characteristics of the turbine. Wind speed at hub height can be calculated by using power-law equation [14]:

$$
V_{\mathrm{z}}=V_{\mathrm{i}}\left(\frac{Z}{Z_{\mathrm{i}}}\right)^{x}
$$

where $V_{\mathrm{z}}$ and $V_{\mathrm{i}}$ are the wind speed at hub and reference height $Z$ and $Z_{\mathrm{i}}$, and $x$ is power-law exponent.

The power output $P_{w}\left(\mathrm{~kW} / \mathrm{m}^{2}\right)$ from wind turbine generator can be calculated as follows [14]:

$$
\begin{array}{ll}
P_{\mathrm{w}}=0, & V<V_{\mathrm{ci}} \\
P_{\mathrm{w}}=a V^{3}-b P_{\mathrm{r}}, & V_{\mathrm{ci}}<V<V_{\mathrm{r}} \\
P_{\mathrm{w}}=P_{\mathrm{r}}, & V_{\mathrm{r}}<V<V_{\mathrm{co}} \\
P_{\mathrm{w}}=0, & V>V_{\text {co }}
\end{array}
$$

where $a=P_{r} /\left(V_{r}^{3}-V_{c i}^{3}\right), b=V_{c i}^{3} /\left(V_{r}^{3}-V_{c i}^{3}\right), P_{r}$ is the rated power, $V_{c i}, V_{c o}$ and $V_{r}$ are the cut-in, cut-out and rated speed of the wind turbine.

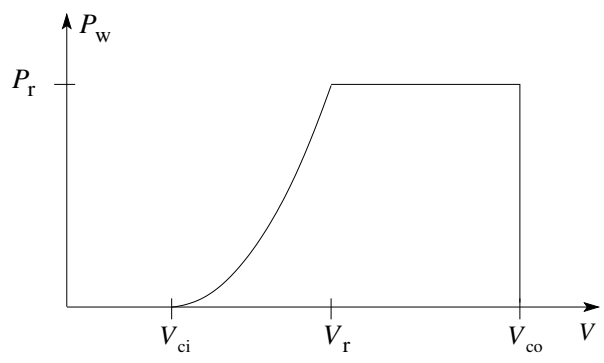

Fig. 6. Power output

In [15] a combined wind simulator and wind power generator model was developed to show agent based capabilities in multiscale simulation. This model is able to simulate wind speed from real time requirements up to simulations on mid time scales using hourly mean values. For this case, the detailed model is used as real time wind speed has to be considered in the microgrid model.

Using historical wind speeds from a specific site, future hourly data can be simulated using the time series model. The simulated hourly mean wind speed can be obtained by

$$
v_{h}(t)=\mu_{h}+\sigma_{h} \cdot y_{t}
$$

where $\mu_{h}$ is the observed hourly mean wind speed and $\sigma_{h}$ the hourly standard deviation. The method is explained in detail in [15] and [16].

The time series model of $y_{t}$ is based on an ARMA (AutoRegressive Moving-Average) model which is given by

$$
\begin{aligned}
y_{t}= & \phi_{1} y_{t-1}+\phi_{2} y_{t-2}+\ldots+\phi_{n} y_{t-n} \\
& +\alpha_{t}+\theta_{1} \alpha_{t-1}+\theta_{2} \alpha_{t-2}+\ldots+\theta_{m} \alpha_{t-m}
\end{aligned}
$$

A data series is used for $y_{t}$ to build the model, i.e. to calculate the auto-regressive $\phi_{i} i=1,2, \ldots, n$ and the moving average parameters $\theta_{j} j=1,2, \ldots, m .\left\{\alpha_{t}\right\}$ is a Gaussian white noise process with zero mean and standard deviation of $\sigma_{\alpha}$.

After having simulated the hourly means, a real time model is used to recreate the turbulent phenomena on this slow varying part. The so called fast term is modeled by the following differential equation that simulates the highly fluctuating signal:

$$
\frac{d w}{d t}(t)=-\frac{w(t)}{T}+\kappa v_{h}(t) \sqrt{\frac{2}{T}} \xi(t)
$$

where $T=L / \bar{v}$, being $L$ the turbulence length scale, $\kappa$ a factor that depends on the geographical location of the wind turbine site, $\xi(t)$ a Gaussian white noise and $v_{h}(t)$ the hourly mean wind speed. The equation describes a stationary Gaussian process.

Having gained the wind speed, the wind generator model described above determines at each time step the power output of the wind generator, that is used to feed into the microgrid. This power is delivered to the corresponding PV-bus. 
3) Photovoltaic $(P V)$ panel: The solar radiation on an inclined plane is given by [14]:

$$
I_{T}=I_{b} R_{b}+I_{d} R_{d}+\left(I_{b}+I_{d}\right) R_{r},
$$

where $I_{b}$ and $I_{d}$ are direct normal and diffuse solar radiations, $R_{d}$ and $R_{r}$ are the tilt factors for the diffuse and reflected part of the solar radiations. $T_{T}$ depends on position of sun in the sky, which varies from month to month.

Hourly power output from PV system with an area $A_{p v}\left(\mathrm{~m}^{2}\right)$ on an average day of jth month, when total solar radiation of

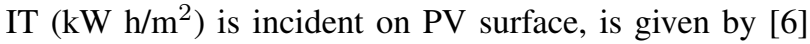

$$
P_{s j}=I_{T_{j}} \eta A_{P V}
$$

where system efficiency $\eta$ is given by

$$
\eta=\eta_{m} \eta_{p c} P_{f}
$$

and the module efficiency $\eta_{m}$ is given by

$$
\eta_{m}=\eta_{r}\left[1-\beta\left(T_{c}-T_{r}\right)\right]
$$

where $Z_{r}$ is the module reference efficiency, $Z_{p c}$ is the power conditioning efficiency, $P_{f}$ is the packing factor, $\beta$ is the array efficiency temperature coefficient, $T_{r}$ is the reference temperature for the cell efficiency and $T_{c}$ is the monthly average cell temperature, calculated as

$$
T_{c}=T_{a}+\frac{\alpha \tau}{U_{L}} I_{T},
$$

where $T_{a}$ is the instantaneous ambient temperature, $U_{L} / \alpha \tau=$ $I_{T, \mathrm{NOCT}} /\left(\mathrm{NOCT}-T_{a, \mathrm{NOCT}}\right)$, and NOCT is normal operating cell temperature, $T_{a, \mathrm{NOCT}}=20^{\circ} \mathrm{C}$ and $I_{\mathrm{T}, \mathrm{NOCT}}=$ $800 \mathrm{~W} / \mathrm{m}^{2}$, for a wind speed of $1 \mathrm{~m} / \mathrm{s}$. Having a known output power and tension, the photovoltaic cell is modeled as a PVbus.

4) Battery storage system: Storage systems comprehend a main part in microgrid networks. Storage elements are fundamental when fluctuating energy sources are used in the grid, as a temporary displacement between the actual generation and consumption exists. As we have to assure that the power injected to the grid matches exactly the demand at any time to assure stability, the introduction of not dispatchable sources like wind or sun leads to a balancing problem. This is specially the case when the fluctuating sources hold a high penetration rate and thus cannot be seen further as negative consumers (by being neglected including them virtually to the demand as a reduction of it). In this case, as a control of the injected renewable energy is hardly possible, a "passiveexternal" control in the sense of storing superfluous energy when demand is satisfied and release it when this is not the case is needed. In the model, the implemented battery storage was modeled as an energy storage device. The storage is sized to meet the load demand during non-availability period of renewable energy source. The battery was connected to the same bus as the PV panel, as it is has become common to install these type of device together as they complement each other. The required battery capacity in ampere hour is given by

$$
B_{\mathrm{rc}}=\frac{E_{\mathrm{c}(A h) D s}}{(D O D)_{\max } \eta_{t}},
$$

where $E_{\mathrm{c}(\mathrm{Ah})}$ is the load in ampere hour, $D_{\mathrm{s}}$ is the battery autonomy or storage days, $\mathrm{DOD}_{\max }$ is the maximum battery depth of discharge, $\eta_{t}$ is the temperature correction factor.

The charge quantity of the battery bank at the time $t$ can be calculated by

$E_{\mathrm{B}}(t)=E_{\mathrm{B}}(t-1)(1-\sigma)+\left(E_{\mathrm{GA}}(t)-E_{\mathrm{L}}(t) / \eta_{\text {inv }}\right) \eta_{\text {battery }}$

where $E_{\mathrm{B}}(t)$ and $E_{\mathrm{B}}(t-1)$ are the charge quantities of battery bank at the time $t$ and $t-1, \sigma$ is the hourly self-discharge rate, $E_{\mathrm{GA}}(t)$ is the total energy generated by renewable energy source after energy loss in controller, $E_{\mathrm{L}}(t)$ is load demand at the time $t, \eta_{\text {inv }}$ and $\eta_{\text {battery }}$ are the efficiency of inverter and charge efficiency of battery bank.

Charge quantity of battery bank is subject to the following constraints:

$$
E_{\mathrm{B}_{\mathrm{m}} \text { in }} \leq E_{\mathrm{B}}(t) \leq E_{\mathrm{B}_{\max }}
$$

where $E_{\mathrm{B}_{\max }}$ and $E_{\mathrm{B}_{\min }}$ are the maximum and minimum charge quantity of battery bank.

Like the other generation sources, the battery is modeled as a PV-bus.

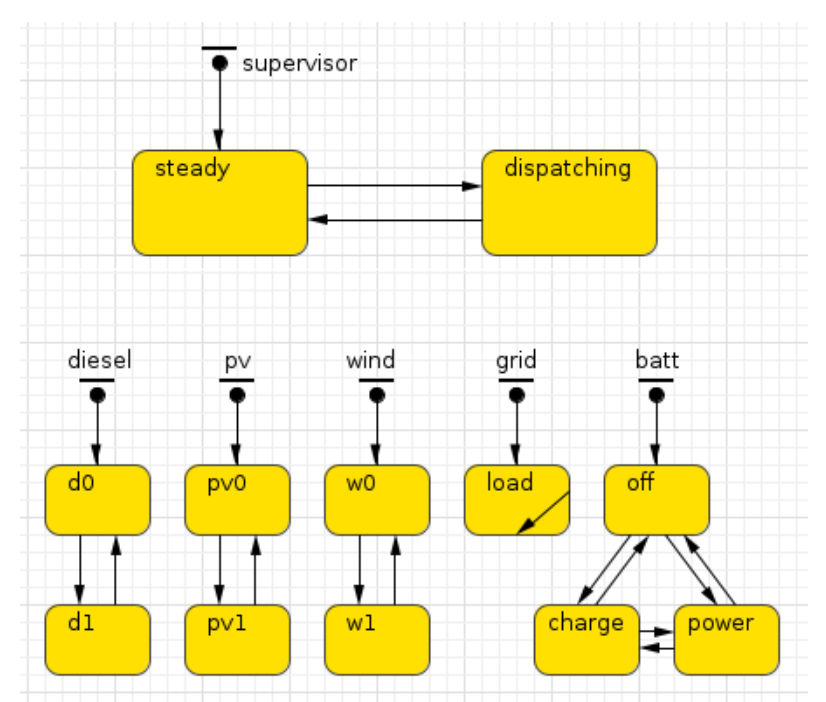

Fig. 7. Supervisor control

5) Load modeling: The modeling of loads can be a quite complex issue. The behavior of a load mainly relies on the electrical energy consumption created by a device. Considering a load in the traditional, electrical engineering sense, a single impedance with some fixed characteristics is meant. But regarding microgrids, the connected loads normally will be households or some other complex consumers that consist of a large number of devices. These devices create an aggregated energy demand that is influenced by each one of the devices. The modeling and simulation of such a demand is a wide topic where still a lot of questions have to be solved. Analyzing 


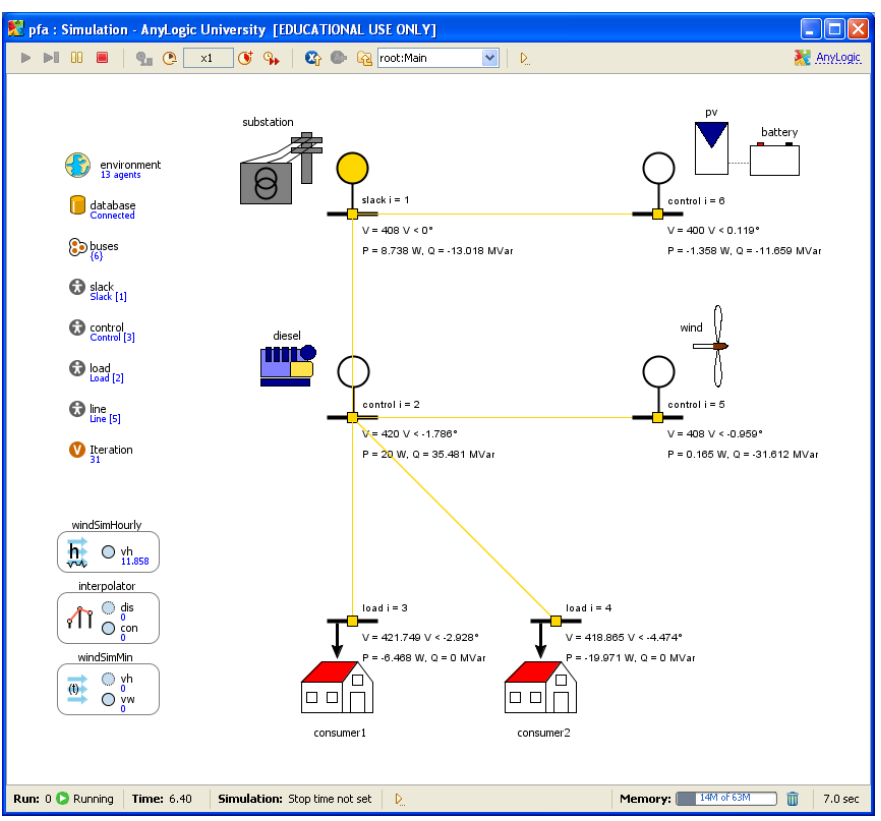

Fig. 8. Simulation view: Power Flow

the load curves of typical households, patterns can be recognized. There are normally some increasing demand peaks in the morning, noon and evening. This peaks are specially pronounced during weekdays, as they coincide with the usage times of most of the high power devices. For representing such a type load in the model, the consumer agents were simplified and a typical curve was assumed. This curve represents an active power demand (almost no reactive power is consumed by households). Thus, the consumer is modeled as a PQ-bus, where the active $P$ and reactive $Q$ power values are known.

6) Substation: Electrical substations are used for power transfer between different levels of the grid. The substation serves mainly as power compensation in order to exchange power with the grid and can be considered as an infinite bus. The simplest substation of this type consists in a transformer. The model can be realized according to the ideal transformer equation

$$
P_{\text {in }}=V_{\text {pri }} \cdot I_{\text {pri }}=P_{\text {out }}=V_{\text {sec }} \cdot I_{\text {sec }}
$$

where $P_{\text {in }}$ and $P_{\text {out }}$ are the powers, $V_{p r i}$ and $V_{\text {sec }}$ the voltages and $I_{p r i}$ and $I_{s e c}$ the currents on the primary and secondary winding, respectively. Using an equivalent circuit for a real transformer, the losses can be included, such as winding resistances, hysteresis losses, etc. The substation is modeled as the slack bus, that means that the complex voltage value is fixed, normally the magnitude to the base voltage value of the per unit system, and the voltage angle is set to zero. It corresponds to the Point of Common Connection (PCC).

\section{Simulation RESUlts}

Once all the objects had been created it is possible to connect them and run simulation. Figure 8 represents the
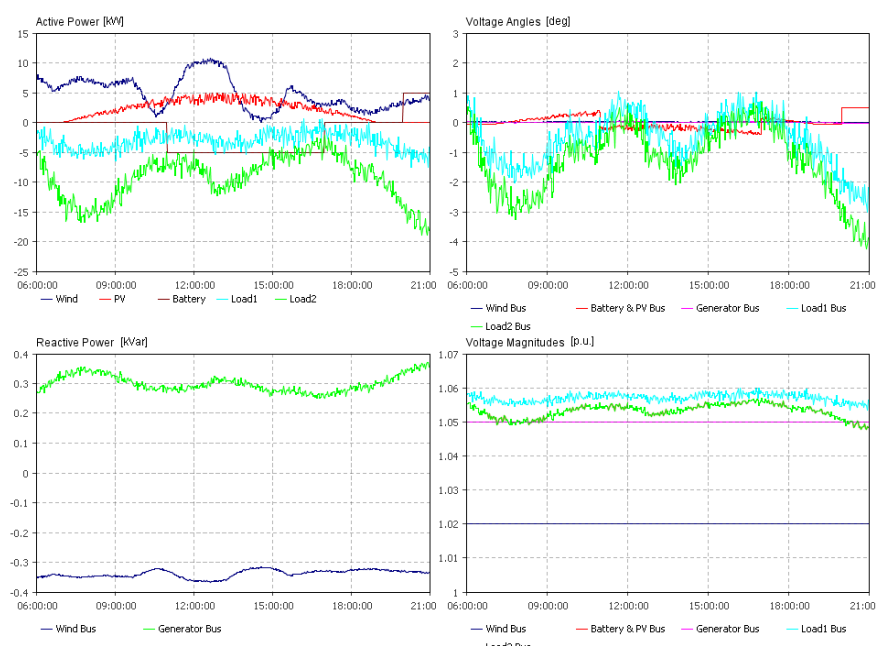

voltage mag

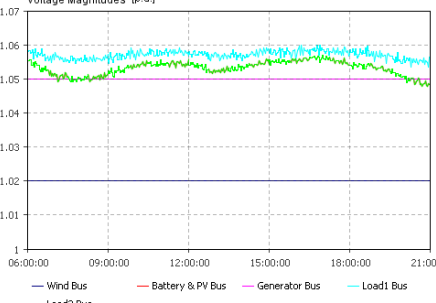

Fig. 9. Simulation view: Graphics results for power and voltage

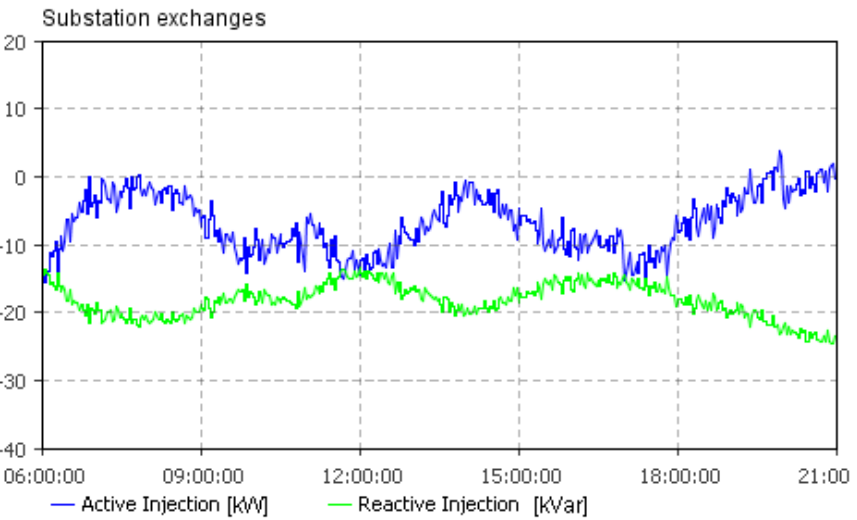

Fig. 10. Simulation view: Graphics results for substation

AnyLogic simulation window of a simple example with a substation (substation), a battery accumulator (battery), a diesel generator (diesel) a wind turbines (wind), and two consumer (consumer1 and consumer2). The data appearing beside the nodes represent the (simulation time) power flow data: power and voltage levels at each bus.

This example shows the microgrid running in connected mode. In this case, the diesel generator is assumed to run at a constant (economically feasible) level. Exceeding and needed power is exchanged with the high tension grid through the substation. The active and reactive power flows can be observed at Figure 10, where positive values represent a flow into the microgrid (shortage operating mode) and negative values an excess of power produced that is absorbed by the transmission grid (surplus operating mode). This is the typical function of the slack bus, regarding the physical layer. The battery is scheduled to charge during the daytime, where the PV panel is supposed to feed in its power. The stored energy is the released at evening and night. The battery storage cycles are assumed on daily cycles.

Figure 9 shows the active and reactive power injections 
of the different buses into the grid, as well as the voltage angles and magnitudes. The randomness of the wind power injection can be observed, as well as the photovoltaic element injection to the grid. The magnitudes of the buses remain relatively stable, as well as the angles for the generation buses. The reactive flows remain small, as there is no reactive consumption on the microgrid.

It can be seen that supply is guaranteed even if load demand of power or meteorological conditions change. This can be made specially illustrative and didactic by letting the user clicking at houses as order to change load demand of each one while historical meteorological conditions also change. In this way it is possible to study the microgrid behavior in all possible conditions which could be very interesting in order to size microgrid elements and design its structure.

Figure 8 shows some graphical results.

\section{CONCLUSION}

An agent based model for simulation of microgrids has been implemented using AnyLogic. The model offers a clear two layer structure, which allows representing both physical and logical interactions between the elements. The logical layer offers a robust base to implement agent communication in real time. The physical layer provides the technical results of the power flow calculation integrated in the model. This allows to compute real time simulations of the grid, which can provide valuable information before having implemented the real grid.

The model is mainly intended to design and test microgrids and it can be used as a tool for design, development and demonstration of control strategies specially Centralized Supervisor Control and Decentralized Load-Dispatch Control, design and demonstration of microgrid operation strategies, design and trying of microgrid communication buses, microgrid optimal design, and economic benefits demonstration.

\section{REFERENCES}

[1] A.R. Bergen and V. Vittal, Power System Analysis, 2nd ed. Prentice Hall, New Jersey, 2000.

[2] Navigant Consulting. Microgrids Research Assessment: Phase 2 Final Report. Prepared for DOE's Office of Electricity Delivery and Energy Reliability. California, May 2006.

[3] (2009, Nov.) Bundesministerium fuer Wirtschaft und Technologie. Projekt E-Energy. [Online]. Available: http://www.e-energy.de/

[4] (2009, Oct.) European Commission website on Microgrids. [Online]. Available: http://www.microgrids.eu

[5] M.W. Maier. (2009, Oct.) TiAC White Paper: Architecting Principles for Systems of Systems. TiAC Enterprise Architecture Survey. Informational Technology Educational Services Inc.Needham, MA [Online]. Available: http://www.infoed.com/Open/PAPERS/systems.htm

[6] (2006, Jan.) European SmartGrids Technology Platform: Visions and Strategy for Europe's Electricity Networks of the Future. European Commission: Directorate-General for Research. EUR 22040, 2006 [Online]. Available: http://ec.europa.eu/research/energy/pdf/smartgrids_en.pdf

[7] Agent-based model. From Wikipedia, the free encyclopedia http://en.wikipedia.org/wiki/Agent-based_model

[8] Northeast Blackout of 2003. From Wikipedia, the free encyclopedia http://en.wikipedia.org/wiki/Northeast_Blackout_of_2003.

[9] $(2009$, Nov.) XJ Technologies website. [Online].Available: http://www.xjtek.com/

[10] Y.G. Karpov, R.I. Ivanovski, N.I. Voropai, D.B. Popov, "Hierarchical Modeling of Electric Power System Expansion by AnyLogic Simulation Software", Power Tech, 2005 IEEE Russia, 27-30 June 2005 pp. 1 - 5.
[11] J.M. González de Durana, O. Barambones, E. Kremers, and P. Viejo, "Complete agent based simulation of Mini-Grids. The Ninth IASTED European Conference on Power and Energy Systems", The Ninth IASTED European Conference on Power and Energy Systems, EuroPES 2009, Palma de Mallorca, Spain, September 7-9, 2009.

[12] J.M. González de Durana, O. Barambones, Object oriented simulation of Hybrid Renewable Energy systems focused on Supervisor Control. 14 ${ }^{\text {th }}$ IEEE International Conference on Emerging Technologies and Factory Automation, ETFA2009, September 22-26, 2009, Palma de Mallorca, Spain.

[13] T. Minagawa, Y. Ichikawa, M. Sato, Y. Ishihara, "Recognition of coherent swing in power systems and detection of step-out using power and current measured on tie lines", Electrical Engineering in Japan, Volume 119 Issue 4, Pages 22 - 31.

[14] M.K. Deshmukh, S.S. Deshmukh. Modeling of hybrid renewable energy systems. Renewable and Sustainable Energy Reviews 12 (2008) pp. 235249.

[15] E. Kremers, N. Lewald, J. M. González de Durana, O. Barambones, "An agent-based multi-scale wind generation model", The Ninth IASTED European Conference on Power and Energy Systems, EuroPES 2009, Palma de Mallorca, Spain, September 7-9, 2009.

[16] R. Billinton, H. Chen and R. Ghajar, "Time-series Models for Reliability Evaluation of Power Systems Including Wind Energy", Microelectron. Reliability, Vol.36. No.9. 1996, pp 1253-1261.

[17] E. Ortjohann, O. Omari, R. Saiju, N. Hamsic, D. Morton, "A Simulation Model For Expandable Hybrid Power Systems", University of Applied Sciences S udwestfalen, Division Soest, Laboratory of Power Systems and Power Economics. Internal Report.

[18] F. Valenciaga, P.F. Puleston "Supervisor Control for a Stand-Alone Hybrid Generation System Using Wind and Photovoltaic Energy", IEEE Transactions on Energy Conversion, vol. 20, no. 2, June 2005. 